\title{
Study of the protective effects of cosmetic ingredients on the skin barrier, based on the expression of barrier-related genes and cytokines
}

\author{
Wenyu Ding ${ }^{1} \cdot$ Linna Fan ${ }^{1} \cdot$ Yan Tian ${ }^{2} \cdot$ Congfen $\mathrm{He}^{1}$
}

Received: 17 August 2021 / Accepted: 30 October 2021 / Published online: 19 November 2021

(c) The Author(s) 2021

\begin{abstract}
Background Sensitive skin is the result of a complex process that is closely linked to the damage of the skin barrier. There are no recognized methods for evaluating the efficacy of anti-allergy products.

Methods In this study, a model of skin barrier damage was created by treating $\mathrm{HaCaT}$ cells with $60 \mu \mathrm{g} / \mathrm{ml}$ of sodium dodecyl sulfate for $48 \mathrm{~h}$. The protective effects of nine cosmetic ingredients, including oat extract (S1), on the skin barrier were investigated based on the gene expression levels of aquaporin3 (AQP3), filaggrin (FLG), caspase-14 (CASP14), and human tissue kallikrein7 (KLK7), as well as those of various interleukins (IL) and vascular endothelial growth factor (VEGF).

Results Among the nine ingredients, S1 had a good protective effect on the function of the skin barrier. It promoted the expression of AQP3, FLG, and CASP14, while inhibiting the expression of KLK7 in HaCaT cells, at a concentration of $0.06 \%$. It also maintained IL-6, IL-8, and VEGF at appropriate levels while promoting the proliferation and differentiation of $\mathrm{HaCaT}$ cells.

Conclusions The above indicators allow for the preliminary establishment of a method to evaluate the efficacy of the barrier protection ability of sensitive skin.
\end{abstract}

Keywords $\mathrm{HaCaT} \cdot$ Sensitive skin $\cdot$ Cosmetic ingredients $\cdot$ Safety testing $\cdot$ Skin barrier

\section{Introduction}

Sensitive skin (SS) refers to a state of hyper-reactivity of the skin under physiological or pathological conditions. It can be characterized by subjective symptoms such as burning, tingling, itching, and tightness, with or without objective signs such as erythema, capillary dilation, and desquamation [1]. The development of SS is a complex process involving barrier function, neurological factors, and inflammatory response [2-5], as shown in Fig. 1, wherein it is shown to be associated with high reactivity, poor tolerance, and

Congfen $\mathrm{He}$

congfenhe@126.com

1 Cosmetics Department, College of Chemistry and Materials Engineering, Beijing Technology and Business University, No. 11 Fucheng Road, Haidian District, Beijing 100048, China

2 Air Force Medical Center, PLA, Beijing 100142, China susceptibility to allergy. Impaired skin barrier function is an important cause of skin sensitivity [6].

Aquaporin3 (AQP3) is involved in the uptake and secretion of substances from cells, contributing to the maintenance of elasticity and the repair of damage. Filaggrin (FLG) connects keratin fibers to maintain the flattened shape of keratinocytes and to form the keratin envelope. Caspase-14 (CASP14) is involved in the terminal differentiation of keratinocytes and assists FLG in forming the complete keratinocyte layer [7-10]. Human tissue kallikrein 7 (KLK 7) hydrolyses the bridging protein [11], which acts as a link between keratinocytes, resulting in impaired barrier function. The above four proteins are important for maintaining skin barrier homeostasis, with high expression of KLK 7 triggering severe skin flaking and low expression of the other three proteins, causing incomplete stratum corneum structure and skin barrier damage. The genes of these four proteins are thus known as skin barrier-related genes, and their expression can determine the health of the skin barrier, as well as indirectly indicate the protective or reparative 


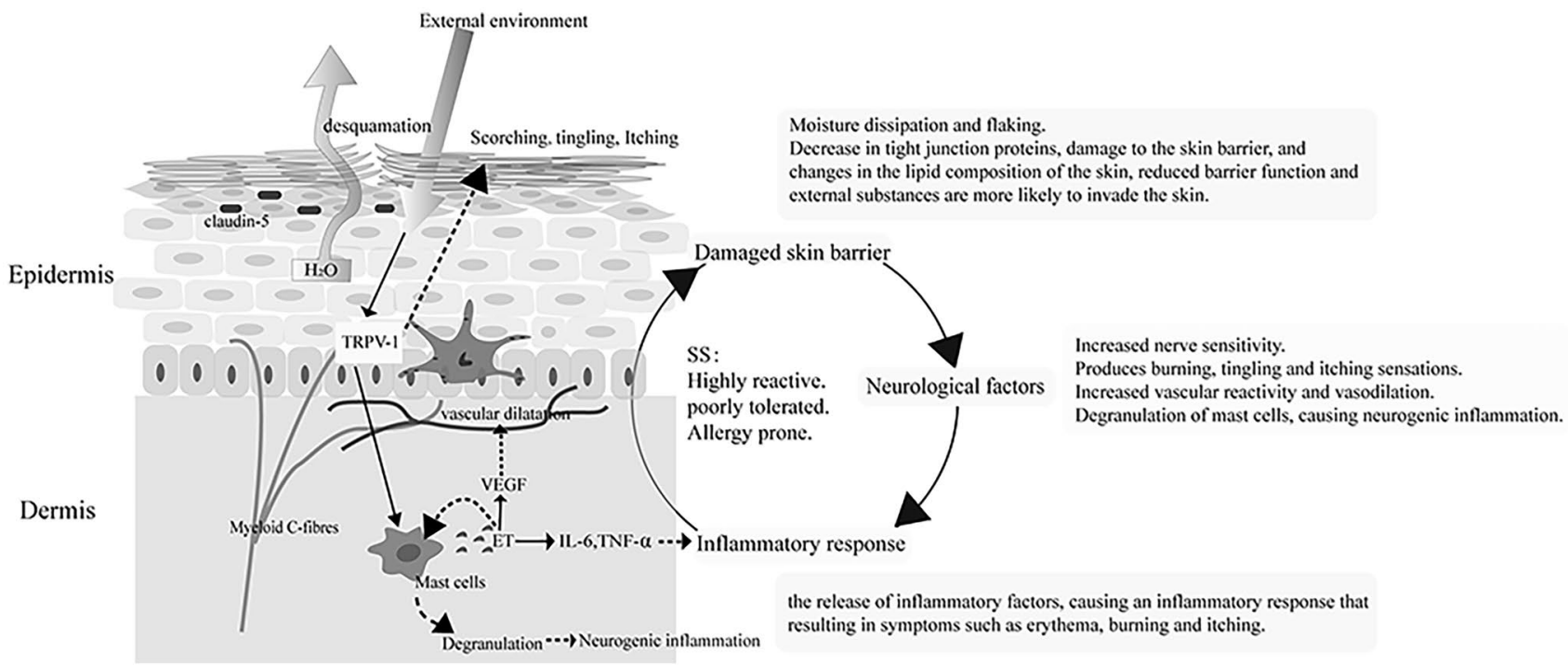

Fig. 1 Causes of skin sensitivity

capacity of a substance tested for its effects on the skin barrier.

The release of interleukin (IL), which is more sensitive to external stimuli, can lead to further damage of the skin barrier. Over-expression of vascular endothelial growth factor (VEGF) induces vasodilation, enhances endothelial permeability, damages blood vessels, and contributes to the development and persistence of inflammation. For these reasons, IL and VEGF are known to be skin barrier-related factors. Measuring the expression of cytokines such as IL can further indicate the extent of damage to the skin barrier, while inhibiting the expression of associated inflammatory factors can mitigate the SS caused by the inflammatory response, as well as reduce its damage to the skin barrier.

In this study, a model of skin barrier damage was created by using SDS to damage $\mathrm{HaCaT}$ cells, which were then used to investigate the protective effect of $\mathrm{S} 1$ and nine other cosmetic ingredients. Levels of AQP3 and those of other indicators were evaluated as key parameters for screening the efficacy of the cosmetic ingredients capable of maintaining the skin barrier. The study aimed to establish a primary experimental system for evaluating the effectiveness and barrier protection ability of sensitive skin.

\section{Material and methods}

\section{Materials}

In this study, nine ingredients that had demonstrated good in vitro biochemical activity in our laboratory, were selected, they have a good ability to scavenge free radicals and inhibit hyaluronidase. They were labeled: oat (Avena sativa) extract
(S1), four stamen stephania (Stephania tetrandra) root (S2), stachyose (S3), erythritol (S4), ceramide 3 liposomes (S5), olive (Olea europaea) leaf extract (S6), compound antiallergic itch-relief remedy (S7/8), and brown algae (Phaeophyceae) extract (S9).

HaCaT (immortalized human epidermal keratinocyte) cells were purchased from the Peking Union Medical College Centre (Beijing, China). DNA markers and real-time PCR components were purchased from Invitrogen Biotechnology (Thermo Fisher Scientific, Waltham, MA, USA). GelRed was obtained from Bio-Rad (Hercules, CA, USA). BD CAB Flex Set was purchased from BD Biosciences (Franklin Lakes, NJ, USA). TRizol was purchased from Ambion (Life Technologies, Carlsbad, CA, USA).

The main instruments employed included: an enzyme labeler (TECAN, Männedorf, Switzerland), a gel imaging analysis system (Alpha Innotech, San Leandro, CA, USA), an Accuri C6 flow cytometer (BD Biosciences), a PCR thermocycler (Eppendorf, Hamburg, Germany), a real-time fluorescence quantitative PCR system (Roche, Basel, Switzerland), and an electrophoresis instrument (Tanon Science $\&$ Technology Co., Ltd., Shanghai, China).

\section{Construction of a HaCaT cell model by SDS}

HaCaT cells were cultured with reference to the method described by Cui et al. [12], and cell concentrations with OD values in the range of 1.0 1.4 were screened by the MTT assay for subsequent experiments. The cells were treated with different concentrations of SDS to determine the appropriate concentration of SDS (cell viability of $60-80 \%$ ) to construct a skin barrier damage model. 


\section{Effect of MTT assay samples on the activity and protection of $\mathrm{HaCaT}$ cells}

Cells were incubated for $48 \mathrm{~h}$ with the sample solutions (experimental group), SDS solution (positive control group), or PBS (blank control group), after which their viability was determined using the MTT assay (Fortuneibo-tech Co., Ltd, Shanghai, China). Six sample concentrations with cell viability above $90 \%$ were selected for subsequent testing. Cells were incubated for $24 \mathrm{~h}$ with the sample solution after which SDS solution was added for another $24 \mathrm{~h}$ of incubation. OD values were measured to detect the protective effect of the samples on the HaCaT cell model. Three sample concentrations (Table 1) with cytoprotective effects were selected for subsequent experiments.

\section{$A Q P 3$ and other barrier-related gene testing}

Real-time fluorescence quantitative PCR was used to evaluate $A Q P 3, F L G, C A S P 14$, and $K L K 7$ gene expression. The experimental systems were all based on the method of CUI et al. [12], and the primer sequences are shown in Table 2.

\section{IL and other cytokine assays}

The effect of the treatment samples on the secretion of IL-6, IL-8, and VEGF by HaCaT cells was measured via cytokine flow assay. According to the instructions of the Human Soluble Protein Master Buffer Kit (BD Biosciences). After the samples were collected (in FCS 2.0 format), a standard curve was plotted and the data analyzed using the Cytometric
Beads Array (CBA) dedicated analysis software, FCAP Array v1.0 (BD Biosciences).

\section{Statistical analysis}

Statistical analysis was performed using IBM SPSS Statistics 22. Each sample was done in 3 parallel. Between-group test using independent samples t-test with one-way ANOVA, $\mathrm{p}<0.05$ indicates a statistically significant difference.

\section{Results}

\section{Construction of a HaCaT cell model of SDS injury}

The MTT method was used to detect the effect of SDS on the activity of $\mathrm{HaCaT}$ cells, and the results showed that SDS was not cytotoxic at concentrations below $30 \mu \mathrm{g} / \mathrm{ml}$, while the cell viability dropped to a minimum above a concentration of $80 \mu \mathrm{g} / \mathrm{ml}$. SDS $(60 \mu \mathrm{g} / \mathrm{ml})$ was chosen as the stimulant concentration in this experiment, and its cell viability was $60 \%$.

\section{Effect of MTT assay samples on the activity and protection of $\mathrm{HaCaT}$ cells}

The nine treatment samples were divided into two categories: liquid samples (S1, S2, S7, and S8) and solid samples (S3, S4, S5, S6, and S9), and the cytotoxicity of each sample was compared after incubation. The results showed that the nine samples were not cytotoxic, some potentially enhancing cell proliferation at low concentrations. Cell viability gradually decreased as the concentration of the samples increased until a point beyond which viability plateaued.

Table 1 The nine samples represented are ingredients, and their specific concentrations, which with cytoprotective effects

\begin{tabular}{llll}
\hline Liquid samples & Low concentration $(\mathrm{v} / \mathrm{v}, \%)$ & Medium concentration(v/v,\%) & High concentration(v/v, $)$ \\
\hline S1 & 0.02 & 0.04 & 0.06 \\
S2 & 0.004 & 0.005 & 0.006 \\
S7 & 0.2 & 0.25 & 0.3 \\
S8 & 0.004 & 0.006 & 0.008 \\
\hline Solid samples & Low concentration $(\mu \mathrm{g} / \mathrm{ml})$ & Medium Concentration $(\mu \mathrm{g} / \mathrm{ml})$ & High concentration $(\mu \mathrm{g} / \mathrm{ml})$ \\
\hline S3 & 200 & 250 & 400 \\
S4 & 300 & 400 & 500 \\
S5 & 200 & 250 & 300 \\
S6 & 100 & 125 & 150 \\
S9 & 0.3 & 0.4 & 0.5 \\
\hline
\end{tabular}

The liquid samples are as follows: S1, oat extract; S2, four stamen stephania extract; S7 and S8, compound anti-allergic itch-relief remedy. The solid samples are as follows: S3, stachyose; S4, erythritol; S5, ceramide 3 liposomes; S6, olive leaf extract; S9, brown algae extract 
Table 2 Primer sequences for real-time fluorescent quantitative PCR

\begin{tabular}{|c|c|c|}
\hline Gene name & & Primer sequences \\
\hline \multirow[t]{2}{*}{ AQP3 } & & Upstream: 5'-AGATGCTCCACATCCGCTAC-3' \\
\hline & & Downstream: 5'-GGTTGATGGTGAGGAAACCA-3' \\
\hline \multirow[t]{2}{*}{ FLG } & & Upstream: 5'-TGACAGTCAGGGACACTCAGA-3' \\
\hline & & Downstream: 5'-GGTGTCTGGAGCCATCTCTT-3' \\
\hline \multirow[t]{2}{*}{ CASP14 } & & Upstream: 5'-CCCAAGGTGTACATCATACAGG-3' \\
\hline & & Downstream: 5'-TCTTTGATGACCATCACAATCTC-3' \\
\hline \multirow[t]{2}{*}{ KLK7 } & & Upstream: 5'-CCTGCTCAGTGGCAATCA-3' \\
\hline & & Downstream: 5'-CAGGTGACAGGTGTACTCAT-3' \\
\hline \multirow[t]{2}{*}{ GAPDH } & House-keeping genes & Upstream: 5'-ACATAGGCGCTCACTGTTCTC-3' \\
\hline & & Downstream: 5'-GCCCAATACGACCAAATCC-3' \\
\hline \multirow[t]{2}{*}{$\beta$-actin } & & Upstream: 5'-CCAACCGCGAGAAGATGA-3' \\
\hline & & Downstream: 5'-CCAGAGGCGTACAGGGATAG-3' \\
\hline
\end{tabular}

\section{Effect of samples on AQP3 gene expression in $\mathrm{HaCaT}$ cells}

SDS $(60 \mu \mathrm{g} / \mathrm{ml})$ significantly reduced the expression of $A Q P 3$ in $\mathrm{HaCaT}$ cells; while samples $\mathrm{S} 1, \mathrm{~S} 5, \mathrm{~S} 6$, and $\mathrm{S} 8$ promoted $A Q P 3$ expression in $\mathrm{HaCaT}$ cells in a dose-dependent manner (Fig. S1). Within a certain range, the concentrations of these treatment samples were directly proportional to $A Q P 3$ gene expression.

\section{Effect of samples on FLG and CASP14 gene expression in $\mathrm{HaCaT}$ cells}

SDS had no significant effect on the expression of $F L G$ gene in $\mathrm{HaCaT}$ cells, but significantly inhibited the expression of the CASP14 gene (Fig. S2). Samples S1, S2, S3, S4, and S5 promoted the expression of $F L G$ and $C A S P 14$ genes, at higher concentrations.

\section{Effect of samples on KLK7 gene expression in $\mathrm{HaCaT}$ cells}

SDS significantly inhibited the expression of $K L K 7$ gene in $\mathrm{HaCaT}$ cells (Fig. S3). Samples S1, S4, S6, S8, and S9 inhibited the expression of $K L K 7$ in $\mathrm{HaCaT}$ cells at high concentrations but promoted it at low concentrations.

\section{Effect of samples on IL-6 secretion by HaCaT cells}

The results of the experiments are shown in Fig. S4. All nine treatment samples promoted IL-6 secretion by $\mathrm{HaCaT}$ cells, but at concentrations below $20 \mathrm{pg} / \mathrm{ml}$. This indicated that IL- 6 could not be stimulated below minimal concentrations, despite the moderate enhancement of the proliferation and differentiation of these cells.

\section{Effect of samples on IL-8 and VEGF secretion by HaCaT cells}

Samples S1, S2, S3, S4, and S5 showed dose-dependent promotion of IL-8 and VEGF secretion by HaCaT cells, and all nine samples showed consistent effects on IL- 8 and VEGF expression (Fig. S5). Small amounts of IL-8 and VEGF promoted the proliferation of $\mathrm{HaCaT}$ cells.

\section{Discussion}

A skin barrier damage model was created in $\mathrm{HaCaT}$ cells by incubating with $60 \mu \mathrm{g} / \mathrm{ml}$ of SDS and the cell survival rate was $60 \%$. This induced changes in skin barrier-related genes and cytokine levels.

Aquaporins (AQP, aquaporin), located in cell membranes, are responsible for the transport of water and small molecules such as glycerol and urea. AQP3 is a member of the family of aquaporins predominant in the skin and play an important role in maintaining skin hydration, as well as regulating the proliferation, migration, and early differentiation of keratinocytes [13, 14]. Studies have shown that AQP3 knockout mice have impaired skin hydration, maintenance of elasticity, and repair of barrier function, and that glycerol administration alleviates this condition [15]. When cells are exposed to inflammatory or high osmolarity conditions, cellular AQP3 expression is upregulated in response to stress, as is the case in aged or atopic dermatitis skin [16-18]. In this study, samples S1, S5, S6, and S8 were found to promote the expression of $A Q P 3$ in a dose-dependent manner; thus, emerging as potentially beneficial for the maintenance of the skin barrier.

The degradation of FLG in the epidermis is a natural process that maintains the moisture content and barrier structure of the stratum corneum. FLG also initiates the degradation 
of keratinocyte nuclei resulting in non-nucleated keratinocytes, hence is important for the terminal differentiation of keratinocytes, and thus the differentiation and formation of the epidermis [19]. CASP14 is a member of the cysteine aspartate-specific protease (caspase protease) family that is specific to the skin, and its activation is closely linked to keratinization of keratinocytes [20]. CASP14 is also a key enzyme for FLG production as it catalyzes the dephosphorylation of pro-FLG in the granular layer of the skin, to form FLG [21]. These processes are essential for regulating the composition of the skin surface barrier [22, 23] and are often used together as an indicator of the functional status of the skin barrier. During instances of external infection or irritation, CASP14 expression is affected and downregulated, leading to a decrease in substances that maintain the skin barrier structure, such as FLG and natural moisturizing factors. This results in impaired barrier function and adverse skin reactions such as dryness and flaking [24]. The effect of the samples used in this study, on the expression of FLG and CASP14 genes in $\mathrm{HaCaT}$ cells was consistent. At higher concentrations, samples S1, S2, S3, S4, and S5 promoted the expression of both FLG and CASP14, while the others had no significant effect on the gene expression of the two proteins. SDS had no significant effect on the secretion of FLG by $\mathrm{HaCaT}$ cells, but significantly inhibited the expression of CASP14, indicating that SDS did not damage HaCaT cells by directly inhibiting the expression of $F L G$ gene, but via inhibiting the expression of CASP14 and thus the formation of FLG.

KLK7, a member of the human tissue kallikreins (KLKs) family, is produced by keratinocytes in the granular layer and secreted into the intercellular spaces of the stratum corneum [25]. KLK7 hydrolyses corneodesmosin and desmocollin, the main components of bridging proteins that connect keratinocytes to each other [11], causing degradation of the bridging proteins and loss of intercellular adhesion, resulting in flaking of the skin. This role of KLK7, in balance with the rate of differentiation of keratinocytes in the granular layer, maintains the vitality and homeostasis of the stratum corneum. However, when KLK7 activity is too high, severe flaking of the skin and impaired barrier function can be triggered. Studies have shown that KLK7 levels are significantly increased in the stratum corneum of irritated [26] or inflamed [27] skin and that topical application of targeted active agents such as salicylic acid [10] can downregulate KLK7 expression and thus alleviate skin barrier damage. In this study, S1, S4, S6, and S9 inhibited KLK7 expression at high concentrations, while the study samples at low concentrations promoted KLK7 expression, indicating that the effective concentration of the active substance should be appropriately increased if the purpose of the cosmetic agent is to maintain the skin barrier.

Damage to the skin barrier and corresponding changes in the gene expression of its associated proteins can stimulate an inflammatory response, causing the release of inflammatory factors. IL-6 is a functionally complex cytokine involved in cell proliferation, differentiation, inflammation, and immune regulation. During pathological conditions, IL-6 overexpression occurs, causing a local inflammatory response and leads to tissue damage [28, 29]. However, during normal physiological conditions, IL-6 is maintained at low levels to stimulate keratinocyte growth, and moderately promote their proliferation and differentiation [30], thereby helping to maintain normal skin metabolism [31]. This study found that addition of nine treatment samples promoted a limited amount of IL-6 secretion in HaCaT cells, but the IL- 6 concentration was below $20 \mathrm{pg} / \mathrm{ml}$, suggesting that the level was low enough not to cause an inflammatory response in an organism.

IL-8 is a chemotactic cytokine that promotes the chemotaxis of lymphocytes and neutrophils, leading to an inflammatory response through a series of actions and is hence considered an important facilitator of inflammation [32]. The small amount of IL-8 expressed in keratinocytes also has a pro-proliferative and auto-chemotactic effect. According to the studies on the expression of IL- 8 in the healing process of human skin tissues after pricking and incision [33] and during skin transplantation in rabbits [34], maintaining a certain amount of IL-8 is important for the healing process after skin injury. Wang et al. [31] observed that when total peony glycosides acted in a concentration range that inhibited IL-8 expression in cell supernatants, they also inhibited cell proliferation, thus indirectly confirming the promotion of keratinocyte proliferation and chemotaxis by the expression of a small amount of IL-8.

VEGF is the most effective factor in promoting the growth of vascular endothelial cells, acting on them by binding to corresponding receptors to promote their proliferation and angiogenesis. The small amount of VEGF expressed in normal skin keratinocytes helps to maintain the normal density and permeability of blood vessels, facilitating nutrient transport and skin metabolism [35]. Overexpression of VEGF has been shown to lead to local vascular hyperplasia and hyper-permeability, which can be used as a biochemical indicator to evaluate the efficacy of drugs in psoriasis [36] and other skin lesions. Contrarily, reducing VEGF levels can effectively diminish its binding to vascular receptors and thus reduce microvascular hyperplasia [37]. In addition, superficial blood vessels are susceptible to external stimuli, which may induce vascular hyper reactivity and release of inflammatory mediators, sequelae closely associated with 
SS [38]. In this study, S5 demonstrated marked promotion of VEGF, which could potentially cause an increase in vascular reactivity and thus SS, implying cosmetics containing this ingredient may be unsuitable for people with SS. Several other ingredients showed weaker stimulation of VEGF, while S6 and S9 inhibited its expression.

\section{Conclusion}

A model for damaged skin barrier was created by using $\mathrm{HaCaT}$ cells exposed to $60 \mu \mathrm{g} / \mathrm{ml}$ SDS. This was used to detect changes in the gene expression of barrier-related proteins-AQP3, FLG, CASP14, KLK7-and cytokines-IL-6, IL-8, and VEGF - which together could be used as indicators for evaluating the efficacy of cosmetic agents in protecting the skin barrier. An ingredient capable of stimulating the expression of $A Q P 3, F L G$, and CASP14, while inhibiting the expression of $K L K 7$, plus maintaining the levels of IL-6, IL-8, and VEGF in the appropriate concentration range, can be important in the protection of the skin barrier. It could be used as a cosmetic ingredient to achieve a soothing effect while maintaining the skin barrier.

Supplementary Information The online version contains supplementary material available at https://doi.org/10.1007/s11033-021-06918-5.

Acknowledgements This work was supported by 2021 Graduate Research Ability Enhancement Program, Beijing Technology and Business University.

Author contributions DWY and FLN performed the experiments, analysed the date and wrote the manuscript; HCF and TY designed the study, supervised the study and revised the manuscript.

\section{Declarations}

Conflict of interest The authors declare that they have no conflict of interest.

Ethical approval This article does not contain any studies with human participants performed by any of the authors.

\section{Informed consent None.}

Consent to participate All authors have confirmed their participation in this study.

Open Access This article is licensed under a Creative Commons Attribution 4.0 International License, which permits use, sharing, adaptation, distribution and reproduction in any medium or format, as long as you give appropriate credit to the original author(s) and the source, provide a link to the Creative Commons licence, and indicate if changes were made. The images or other third party material in this article are included in the article's Creative Commons licence, unless indicated otherwise in a credit line to the material. If material is not included in the article's Creative Commons licence and your intended use is not permitted by statutory regulation or exceeds the permitted use, you will need to obtain permission directly from the copyright holder. To view a copy of this licence, visit http://creativecommons.org/licenses/by/4.0/.

\section{References}

1. Qian T, Hao F (2020) Epidemiology of sensitive skin. Dermatol Bull 37:559-564+2

2. Tu Y, Zhang YJ, He L (2020) Functional cosmetic and sensitive skin. Dermatol Bull 31:607-611+6-7

3. Shu H, He L (2020) Pathogenesis of sensitive skin. Dermatol Bull 37:555-558+551

4. Chen LH, Jiang J (2020) Update of pathogenesis of sensitive skin and related skin diseases. China J Lepr Skin Dis 36:505-508

5. Fan LN, Jia Y, Jiang LG et al (2015) Brief analysis of causes for sensitive skin and progress on evaluation methods for antiallergic activity of cosmetics. China Surfactant Deterg Cosmet 45:409-414

6. Berardesca E, Farage M, Maibach H (2013) Sensitive skin: an overview. Int J Cosmet Sci 35:2-8. https://doi.org/10.1111/j. 1468-2494.2012.00754.x

7. Zhang FF (2013) Effects of BaP and UVB on Caspase-14 and filaggrin expression in human keratinocytes. Tianjin Medical University, Tianjin

8. Irvine AD, McLean WH, Leung DY (2011) Filaggrin mutations associated with skin and allergic diseases. N Engl J Med 365:1315-1327. https://doi.org/10.1056/NEJMra1011040

9. Shan SJ (2009) Regulation of semen coicis extraction on expression of aquaporin 3 induced by ultraviolet B irradiation in $\mathrm{HaCaT}$ cells. China Medical University, Liaoning

10. Zhao D (2012) Effects of Salicylic acid on the expression of filaggrin (FLG) and kallikrein (KLK7) in human keratinocytes. Dalian Medical University, Liaoning

11. Tan X, Yang LT, Sun LD (2015) Kallikrein 7 is a potential target for treatment of atopic dermatitis. Chem Life 35:715-720

12. Cui L (2017) Screening and studying of facial lipid biomarkers for SPSS females by lipidomics. Beijing Technology and Business University, Beijing

13. Huang SY, Tu Y, He L et al (2017) Research progress on the relationship between aquaporin 3 and skin barrier. Dermatol Bull 34:383-387+382

14. $\mathrm{Pu} \mathrm{CX}$ (2010) Research progress on aquaporin. J Chengdu Univ (Natural Science Edition) 29:104-106

15. Zhang JX, Li ZJ (2013) Research progress in role of AQP3 in pathogenesis of skin diseases. Med Recapitul 19:3101-3103

16. Sugiyama Y, Ota Y, Hara M et al (2001) Osmotic stress up-regulates aquaporin-3 gene expression in cultured human keratinocytes. Biochim Biophys Acta 1522:82-88. https://doi.org/10. 1016/s0167-4781(01)00320-7

17. Olsson M, Broberg M, Jernas M et al (2006) Increased expression of aquaporin 3 in atopic eczema. Allergy 61:1132-1137. https://doi.org/10.1111/j.1398-9995.2006.01151.x

18. Liu FF (2013) Mechanistic study of aquaporin 3 in photoaging of normal human skin fibroblasts. Central South University, Changsha

19. Alibardi L, Tschachler E, Eckhart L (2005) Distribution of caspase-14 in epidermis and hair follicles is evolutionarily conserved among mammals. Anat Rec A 286a:962-973. https:// doi.org/10.1002/ar.a.20234

20. Denecker G, Hoste E, Gilbert B et al (2007) Caspase-14 protects against epidermal UVB photodamage and water loss. Nat Cell Biol 9:666-674. https://doi.org/10.1038/ncb1597 
21. Nicotera P, Melino G (2007) Caspase-14 and epidermis maturation. Nat Cell Biol 9:621-622. https://doi.org/10.1007/ s00441-013-1557-2

22. Ginger RS, Blachford S, Rowland J et al (2005) Filaggrin repeat number polymorphism is associated with a dry skin phenotype. Arch Dermatol Res 297:235-241. https://doi.org/10.1007/ s00403-005-0590-8

23. Fischer H, Stichenwirth M, Dockal M et al (2004) Stratum corneum-derived caspase-14 is catalytically active. FEBS Lett 577:446-450. https://doi.org/10.1016/j.febslet.2004.10.046

24. Li J, Chen JP, Wu J (2010) Effects of candida albicans infection on caspase-14 expression in mouse skin. J Diagn Ther Dermatovenereol 17:24-27

25. Cheng F, Guo Y (2014) Relationship between mutations of skin barrier-related genes and atopic diseases. Chin J Dermatovenereol 28:959-962

26. Torma H, Lindberg M, Berne B (2008) Skin barrier disruption by sodium lauryl sulfate-exposure alters the expressions of involucrin, transglutaminase 1, profilaggrin, and kallikreins during the repair phase in human skin in vivo. J Invest Dermatol 128:1212-1219. https://doi.org/10.1038/sj.jid.5701170

27. Lu XY, Li YZ, Men YH (2013) Study on molecular mechanisms of impaired skin barrier function in an atopic dermatitis-like animal model. China J Lepr Skin Dis 29:164-167

28. Wang F, Zhang JX, Xu SH (2014) Expressions of IL-6 in skin and serum of TCE-sensitized mice. China Occup Med 41:14-19

29. Andreozzi F, Laratta E, Procopio C et al (2007) Interleukin-6 impairs the insulin signaling pathway, promoting production of nitric oxide in human umbilical vein endothelial cells. Mol Cell Biol 27:2372-2383. https://doi.org/10.1128/MCB.01340-06

30. Li J, Sun WB, Lv YY (2011) The changes of CC, IL-8/CXCL8 chemokine in the reaction of rhIL-10 resisting the skin graft. Chinese J Immunol 27:1123-1128
31. Wang JM (2013) Effects of total glucosides of paeony on expression of IL-8, ICAM-1 and Ki67 in human HaCaT keratinocytes. Lanzhou University, Gansu

32. Hennus MP, van Vught AJ, Brabander M et al (2013) Mechanical ventilation drives inflammation in severe viral bronchiolitis. PLoS ONE 8:e83035. https://doi.org/10.1371/journal.pone.0083035

33. Huang HL, Nie X, Cai B et al (2013) Procalcitonin levels predict acute kidney injury and prognosis in acute pancreatitis: a prospective study. PLoS ONE 8:e82250. https://doi.org/10.1371/journal. pone. 0082250

34. Tao Y (2013) The effect of gypenosides-containing serum on the p38MAPK signal pathway in photoaging human skin keratinocytes. Liaoning University of Traditional Chinese Medicine, Liaoning

35. Li FF, Mi LG, Sui HJ (2009) Progress of impact of VEGF on vascularization of skin. J Dalian Med Univ 31:599-603

36. Zhang Y (2014) Influence of total glucosides of paeony on the expression of VEGF mRNA in skin tissue and peripheral blood of BALB/c mice psoriasis. Southern Medical University, Guangzhou

37. Xia M, Duan XW, Gao YH et al (2011) Regulatory effects of Xiaoyin Jiedu decoction on tumor necrosis Factor- prosuction in psoriatic patients with blood-heat syndrome. Chin J Dermatovenereol Integr Tradit West Med 10:79-82

38. Jiang WC, Zhang H, Xu Y et al (2020) Cutaneous vessel features of sensitive skin and its underlying functions. Skin Res Technol 26:431-437

Publisher's Note Springer Nature remains neutral with regard to jurisdictional claims in published maps and institutional affiliations. 